\title{
Identification and expression analyses of new potential regulators of xylem development and cambium activity in cassava (Manihot esculenta)
}

Tyche Siebers, 1

Bruno Catarino, 1

Javier Agusti, $1,2,{ }^{*}$

Phone +34963877856

Email jagusti@ibmcp.upv.es

1 Department of Plant Sciences, University of Oxford, South Parks Road, Oxford, OX1 3RB UK

2 Present Address: Instituto de Biología Molecular y Celular de Plantas (CSIC-UPV), C/Ingeniero Fausto Elio s/n, 46011 Valencia, Spain

\section{Abstract}

\section{Main conclusions}

We have identified new potential regulators of xylem cell-type determination and cellular proliferation in cassava and studied their expression in roots. Results are highly relevant for cassava biotechnology.

Cassava's root system is composed of two types of root that coexist in every individual: the fibrous and the storage roots. Whether a root becomes fibrous or storage depends on the xylem cell types that it develops: fibrous roots develop xylem fibres and vessels while storage roots develop parenchyma xylem, the starch-storing tissue. A crucial question in cassava root development is how the specific xylem cell types differentiate and proliferate in the fibrous and storage roots. Using phylogenetic, protein sequence and 
synteny analyses we identified (1) MeVND6, MeVND7.1, MeVND7.2, MeNST3.1 and MeNST3.2 as the potential cassava orthologues of the Arabidopsis regulators of xylem cell type determination AtVND6, AtVND7 and AtNST3; and (2) MeWOX4.1 and MeWOX4.2 as the potential cassava orthologues of the Arabidopsis cambium regulator AtWOX4. Fibrous and storage roots were anatomically characterised and tested for the expression of the identified genes. Results revealed that (1) MeVND7.1 and MeVND7.2 are expressed in the fibrous but not in the storage roots; (2) MeVND6 shows low expression in both root types; (3) MeNST3.1 is not expressed in the fibrous or storage roots, while MeNST3.2 is highly expressed in both root-types and (4) MeWOX4.1 and, to a higher level, MeWOX4.2 are expressed in both the fibrous and storage roots. Results open new avenues for research in cassava root development and for food security-oriented biotechnology programmes.

\section{Keywords}

Cambium

Cell fate

Fibrous root

Meristem

Storage root

Xylem

\section{Electronic supplementary material}

The online version of this article (doi:10.1007/s00425-016-2623-2) contains supplementary material, which is available to authorized users.

\section{Introduction}

By 2050 the world population is expected to reach 9.1 billion, the average global temperature will rise $2-4{ }^{\circ} \mathrm{C}$, hydric resource will be scarce and, as a consequence of the growth of urban areas, the arable land surface will dramatically decline (http://www.fao.org). In such context, improving our main staple crops to guarantee sustainable food security is of top priority, especially in those regions that are already food insecure. Cassava (Manihot esculenta) is the major staple crop in sub-Saharan Africa, where it represents the main source 
of carbohydrates for $\sim 500$ million people. Its ability to accumulate high amounts of starch in its storage root, grow in many types of soil and resist severe drought and high temperature conditions converts cassava into the preferred (and in many cases the only possible) crop for sub-Saharan breeders and self-sustained farmers (Lebot 2009).

Despite of its importance, there are a number of constraints that limit the yields and quality of cassava. For example, cassava's nutritional value is low because, although roots are very efficient in accumulating starch, they are inefficient in accumulating proteins, vitamins or micronutrients (Sayre et al. 2011). In addition, cassava storage roots degrade very quickly after harvesting due to a highly oxidative process known as post-harvest physiological deterioration (PPD) that makes roots inedible in 24-72 h (Reilly et al. 2007). Diseases such as the cassava mosaic virus or the brown streak also result, ultimately, in severe root symptoms (i.e. growth delay or corky texture) (Lebot 2009). Furthermore, biomass availability for food might be threatened in the mid- to long-term, because cassava is increasingly becoming a strategic crop for the bio-energy industry due to its quick and cheap starch production capacities (Saithong et al. 2013 ). In recent years valuable efforts have been pursued to address these constraints through breeding and biotechnology (Sayre et al. 2011; Ceballos et al. 2012; Xu et al. 2013 ). However, to reach these goals with guarantees, it is crucial to shed new light on the molecular regulation of cassava root development (Chaweewan and Taylor 2015; Sojikul et al. 2015).

The cassava root system consists mainly on two types of roots that coexist in every individual: the fibrous (woody) roots and the storage roots (Fig. 1a, b). The fibrous roots transport water and nutrients, while the storage roots store starch and are, thus, the edible part. Initially, all roots are fibrous. However, upon an unknown signal, between three and 14 of these fibrous roots (depending on the cultivar) become storage roots (Alves 2002; El-Sharkawy 2004; Lebot 2009). At the cellular level, such transformation is reflected on the type of secondary xylem cells that each root develops: the fibrous root produces xylem fibres and xylem vessels while the storage root produces almost exclusively xylem parenchyma cells. In such xylem parenchyma cells is where the starch granules biosynthesise and accumulate (Lebot 2009). The molecular control underlying the switch from fibrous to storage root development is a complex question that remains unknown. However, identifying genes regulating 
xylem cell-type identity and unravelling general aspects of their expression pattern in the fibrous and storage roots is the first step towards understanding how xylem cell-type determination participates in the process. Moreover, considering that secondary xylem differentiates from the meristematic cells within the cambium, identifying genes involved in cambium activity and studying their expression pattern in both root types may provide new clues about the regulation of cellular proliferation in each of the root types.

\section{Fig. 1}

The root of cassava. a Root system of cassava. b Schematic view of a. FR fibrous root, $S R$ storage root. Note that fibrous roots transforming to storage roots still maintain a fibrous zone

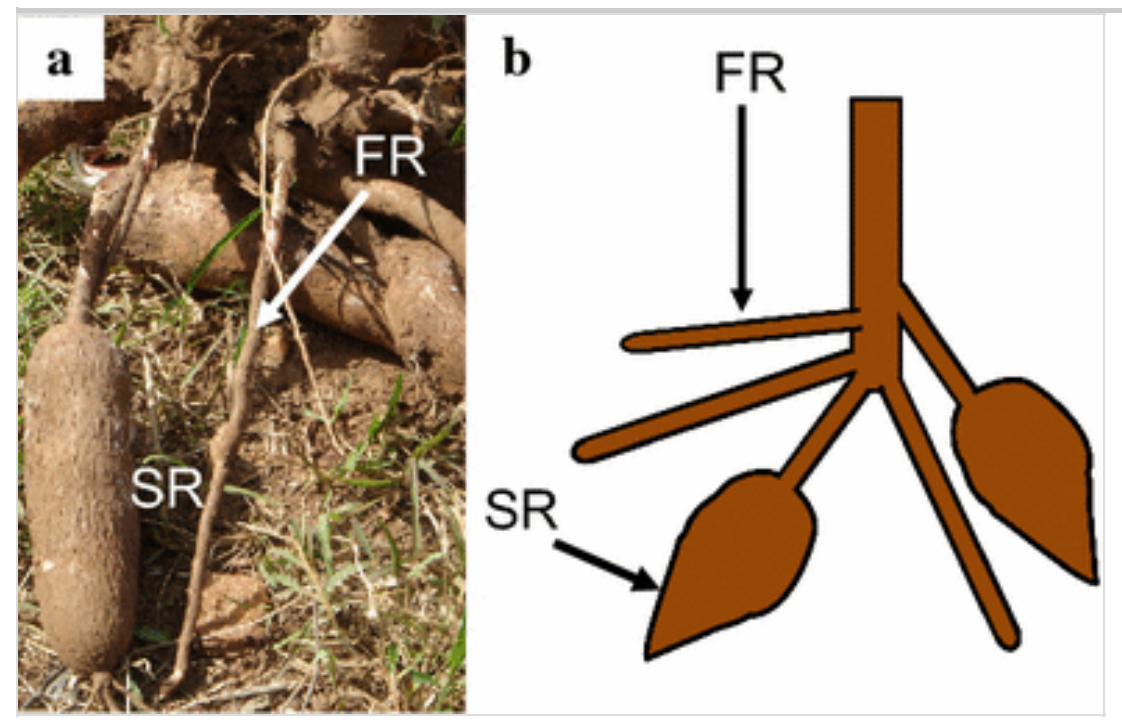

Genetic regulation of cassava root development has barely been explored (Sojikul et al. 2015). However, research in the model system Arabidopsis thaliana has been very prolific in this respect and, indeed, key regulatory genes are known for most of the developmental processes operating in the root, including xylem cell-type differentiation and cambium activity. It is, therefore, possible to take advantage of such knowledge in order to identify orthologues for these regulators in cassava and study their expression patterns in fibrous and storage roots.

In Arabidopsis, the VND6, VND7 and NST3/SND1 genes belong to the NAC family of transcription factors (Olsen et al. 2005), and are members of the VASCULAR-RELATED NAC-DOMAIN (VND) and NAC SECONDARY 
WALL THICKENING PROMOTING FACTOR/SECONDARY WALLASSOCIATED NAC DOMAIN PROTEIN (NST/SND) subclasses. VND6, VND7 and NST3/SND1 are, indeed, the transcription factors acting as first level master switches of xylem cell differentiation (Ohashi-Ito et al. 2010; Nakano et al. 2015) with VND6 and VND7 regulating vessel differentiation (Kubo et al. 2005) and NST1 and NST3/SND1 regulating xylem fibre cell differentiation (Mitsuda et al. 2005, 2007; Zhong et al. 2006). VND6 and VND7 play a key role in vessel development by regulating programmed cell death (PCD; the final step in the process) (Ohashi-Ito et al. 2010). NST3 upregulates the expression of a number of genes encoding MYB-type transcription factors controlling the biosynthesis of major secondary cell wall components (cellulose, xylan and lignin) (Zhong et al. 2008; Ohashi-Ito et al. 2010). Remarkably, overexpression of NST3 in Arabidopsis induces secondary cell wall thickening in epidermis and in the parenchyma cells of leaves and floral organs, but not in the parenchyma of any other organ, indicating either that parenchymatic cells in different organs are differently sensitive to NST3mediated secondary cell wall thickening or that NST3 can be degraded in a cell type-specific manner (Zhong et al. 2006).

WUSCHEL-RELATED-HOMEOBOX 4 (WOX4) is a transcription factor that positively regulates cellular proliferation in the cambium in Arabidopsis (Hirakawa et al. 2010; Ji et al. 2010; Suer et al. 2011). WOX4 is specifically expressed in the procambium and cambium of both roots and shoots, and wox4 mutants display reduced secondary growth (Ji et al. 2010; Hirakawa et al. 2010; Suer et al. 2011).

In this study we have (1) identified the putative cassava orthologues of VND6, $V N D 7, N S T 3$ and WOX4 through phylogenetic, protein sequence and synteny analyses (2) studied the expression patterns of such genes in samples enriched in cambium and developing xylem of fibrous or storage roots. In addition, we have conducted an anatomical study of the fibrous and storage sections of the root with the aim of visualising and confirming anatomical differences between the fibrous and the storage root and correlating such differences with the gene expression profile of all the analysed genes in each root type. We provide evidences of differential molecular regulation during fibrous or storage root development. Results can be exploited for biotechnology applications to improve cassava's yields and properties as a food resource in the future. 


\section{Materials and methods}

\section{Construction of phylogenetic trees}

Phylogenetic trees were constructed for NAC and WOX proteins (cf Fig. 2) as follows. We first performed BLAST (Altschul et al. 1990) searches against the cassava genome (http://phytozome.jgi.doe.gov/pz/portal.html) using the NAC or WOX Arabidopsis protein sequences. All similar cassava protein sequences identified were used. Sequences were then aligned using MAFFT (Katoh and Standley 2013). The alignment was converted to a FASTA format and trimmed using BioEdit (Ibis bioscience). The trimmed alignment was uploaded to ATCG PhyML 3.0 (Guindon et al. 2010) to generate a tree using the following parameters: substitution model L, type of tree improvements NNI, fast likelihood based method aLRT SH-like. We used the sequence for Arabidopsis WOX11 and the corresponding cassava sequence as a known outgroup for the WOX phylogenetic tree based on previous information about WOX proteins (Graaff et al. 2009). For the NAC tree, AtNAC100, which is known to be in a different NAC sub-group, was used to create an outgroup (Hussey et al. 2015).

Fig. 2

Identification of putative cassava genes. a Phylogenetic analyses of NAC transcription factors between Arabidopsis and cassava identify the potential VND6, VND7 and NAC3 orthologues in cassava (note that AtNAC101 is AtVND6, MeNAC009 is MeVND6, AtNAC012 is AtVND7, MeNAC015 is MeVND7.1, MeNAC005 is MeVND7.2, AtNAC030 is AtNST3, MeNAC010 is MeNST3.1 and MeNAC003 is MeNST3.2). b Phylogenetic analyses of WOX transcription factors between Arabidopsis and cassava identify the potential WOX4 orthologues in cassava (note that, based on this tree, in this work MeWOX11 has been renamed to MeWOX4.2 and MeWOX5 to MeWOX4.2) 
a

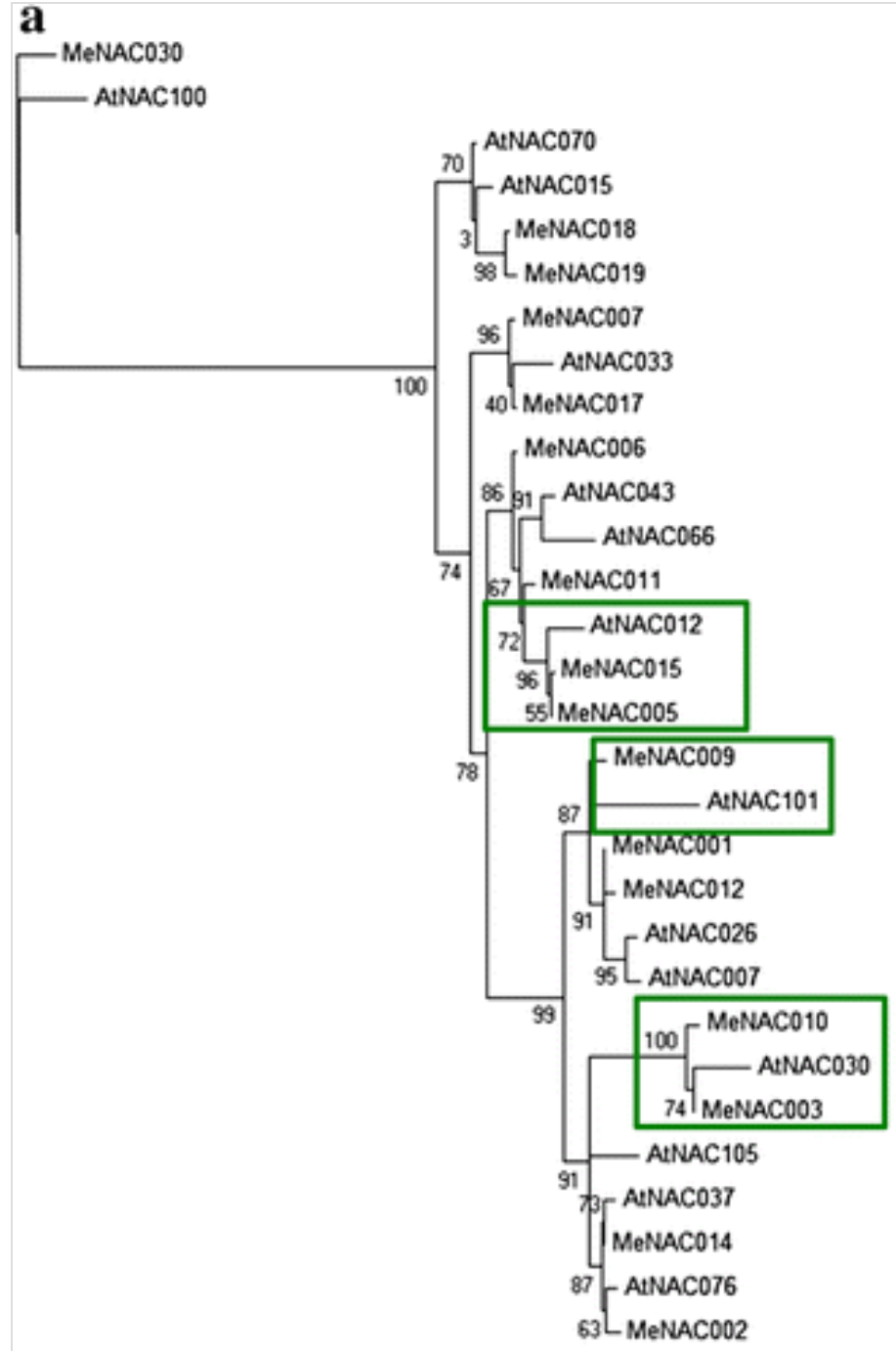

b

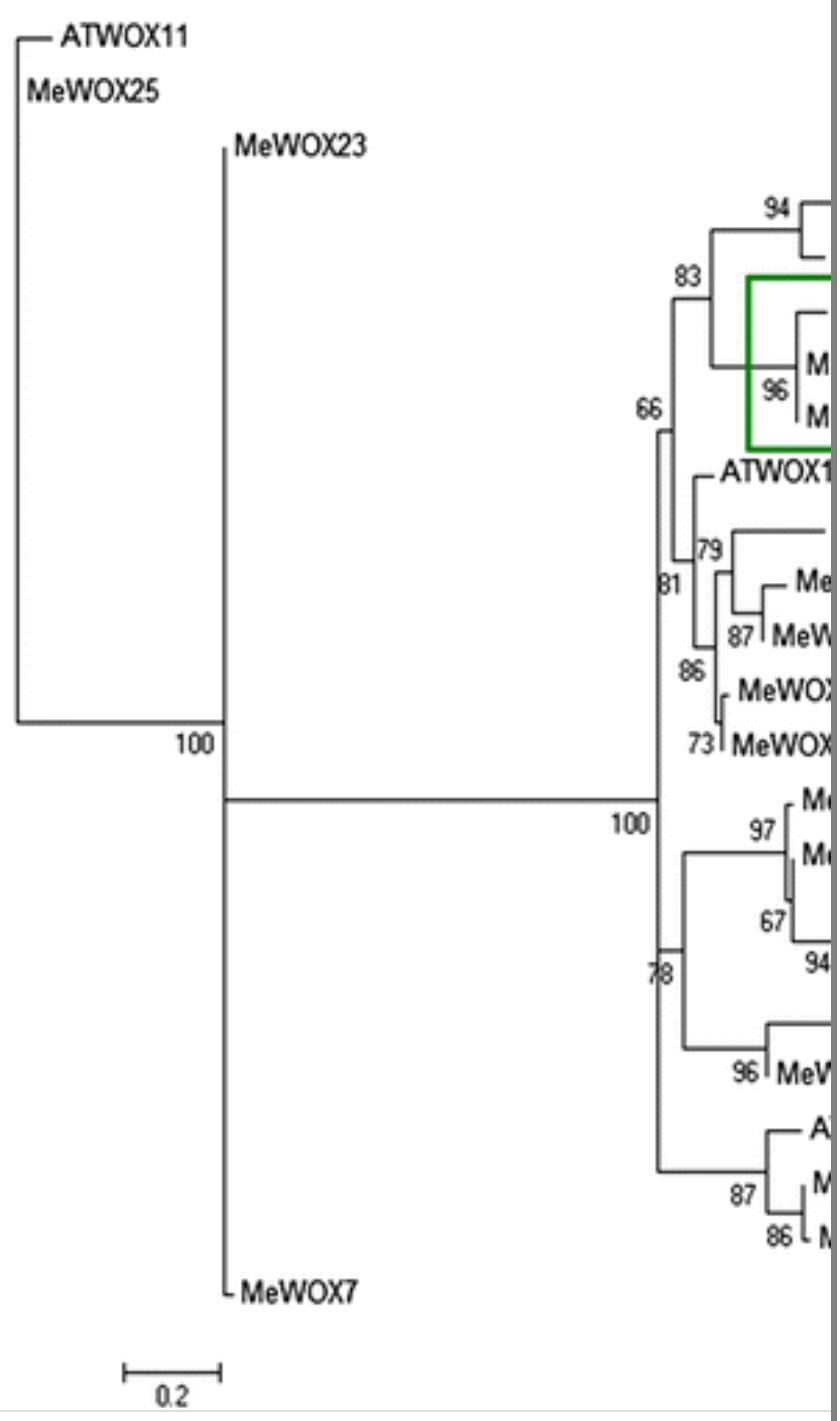

\section{Sequence and synteny analysis}

MEME software (Bailey et al. 2009) was used to compare the sequences of the most likely cassava orthologues of VND6, VND7, NST3 or WOX4 (based on the phylogenetic tree) and to identify conserved domains. InterProScan 5 (Jones et al. 2014 ) was used to confirm the presence of the WOX and NAC domains. Synteny analyses were performed using an available built-in tool in phytozome (https://phytozome.jgi.doe.gov/pz/portal.html\#).

\section{Tissue collection for RNA extraction and histological staining}

Cassava plants of cultivar 60,44460444 were kindly provided by Dr. John Beeching (Bath University). Plants were grown from stem cuttings for 6 months before the start of the experiment to ensure proper fibrous root and storage root 
development. Standard growth conditions were $28{ }^{\circ} \mathrm{C}$ and long-day $(16 / 8$ light/dark) regime. Plants were watered twice a week until the first leaves emerged and thereafter once a week. Plants were quickly removed from pots and, briefly, we collected roots by removing them from the plant as close to the base of the stem as possible. All samples consisted on roots in which the fibrous and the storage root zone were clearly differentiable (cf Fig. 3 a). After collection, roots were quickly washed with water to remove excess soil. For histological analyses, a 0.5 -cm-long section of each root type (fibrous or storage) was put into a Falcon tube containing 4\% paraformaldehyde (PFA) and preserved for wax embedding and tissue staining. For RNA extraction, samples enriched in cambium and developing xylem were dissected free hand from the roots using a razor blade and forceps, and immediately frozen in liquid nitrogen (due to the large size of cassava roots, the cambium and developing xylem zones are visible with naked eye). Samples were then stored at $-80{ }^{\circ} \mathrm{C}$ until needed.

\section{Fig. 3}

Cassava root anatomy. a Global view of a cassava root containing both, the fibrous root (FR) zone and a storage root (SR) zone. b, c Toluidine blue stains on histological sections of the fibrous root (b) or the storage root (c). $C$ cambium, $C X$ cortex, $P$ phellem, $P H$ phloem, $X$ xylem. Scale bars $1 \mathrm{~cm}(\mathbf{a}), 25 \mu \mathrm{m}(\mathbf{b}, \mathbf{c})$ 


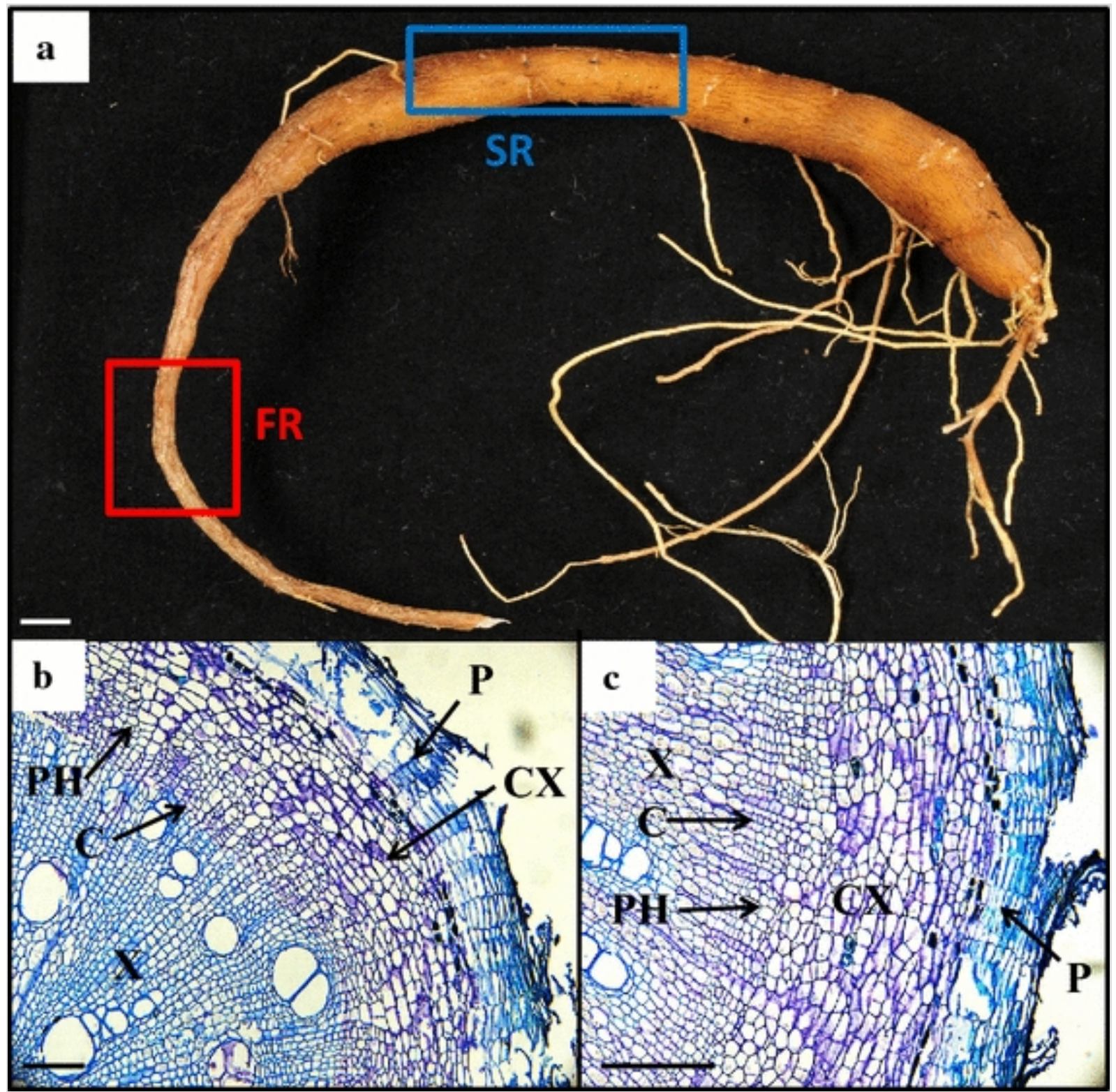

\section{Wax embedding, slide preparation, stains and samples observation}

Samples were embedded using a Tissue-Tek ${ }^{\circledR} \operatorname{VIP}^{\mathrm{TM}}\left(\right.$ By Sakura $\left.^{\circledR}\right)$ vacuum infiltration, embedded in wax using a Tissue-Tek ${ }^{\circledR}$ TEC $^{\text {TM}}\left(\right.$ By Sakura $\left.{ }^{\circledR}\right)$ machine, sectioned and mounted as described (Sehr et al. 2010; Agusti et al. 2011). Toluidine blue stain, and phloroglucinol stain were conducted as previously described (Agusti et al. 2008, 2011; Sehr et al. 2010). For iodine staining a $2 \%(\mathrm{w} / \mathrm{v})$ solution of potassium iodide was prepared and used to stain slides containing fibrous or storage roots. The reaction was prolonged for 10 min. An Olympus BX50 microscope and the program Image Pro Plus were used to photograph all the slides. All photographs were taken at $10 \times$ or $20 \times$ magnification. 


\section{RNA extraction, cDNA synthesis, primer design and gene expression analyses}

Plant material was finely ground on liquid nitrogen using a mortar and pestle. Total RNA extraction was carried out using the Trizol protocol, as previously described (Sehr et al. 2010). RNA was DNase treated using DNase I (Invitrogen ${ }^{\mathrm{TM}}$, 18068-015). Extracted RNA sample quantity and quality were assessed using a NanoDrop ${ }^{\circledR}$ ND-1000 spectrophotometer (Thermo Scientific). cDNA was synthesised using 697.4 ng of RNA (the maximum possible given the RNA sample quantities and limit of the cDNA reaction volume) cDNA synthesis was performed using the RevertAid H Minus First Strand cDNA synthesis kit (Thermo Scientific), following the manufacturers protocol. The cDNA was stored at $-80{ }^{\circ} \mathrm{C}$ until used. Primers were designed using the Primer3Plus program (Untergasser et al. 2007) as described (Agusti et al. 2008 ). Previously validated reference genes for cassava (Whankaew et al. 2015 ) were used. Specificity of the primers was confirmed by running BLAST against the whole cassava genome. All primer sequences are available in Table S1. PCR was performed on the cDNA to assess expression levels, as described (Sehr et al. 2010). After amplification samples were loaded into a 2.2\% agarose gel, containing $2 \%$ sybr safe DNA dye, and electrophoresed at $120 \mathrm{~V}$. Results were visualised using a BioRad UV transilluminator. Three biological replicates, and three technical replicates were used for each gene (i.e. each biological replicate was tested 3 times per gene). Each biological replicate consisted on material coming from 3 different plants.

\section{Results}

Phylogenetic, protein and synteny analyses identify the putative cassava orthologues of VND6, VND7, NST3 and WOX4

In Arabidopsis, VND6, VND7, NST3 and WOX4 are transcription factors belonging to the NAC and WOX families, respectively. To identify the most likely orthologues for such proteins in cassava, we constructed maximum likelihood phylogenetic trees for NAC and WOX proteins. Our phylogenetic analyses (Fig. 2 a, b, Supplementary Table S2; Supplementary Files S1 and S2) identified two potential orthologues for VND7, NST3 and WOX4, respectively, 
and one for VND6. Accordingly, we named the identified proteins MeVND6 (MeNAC009/Manes.06G135700.1), MeVND7.1

(MeNAC010/Manes.18G100800.1), MeVND7.2

(MeNAC003/Manes.02G191900.1), MeNST3.1

(MeNAC015/Manes.02G001600.1), MeNST3.2

(MeNAC005/Manes.01G055400), MeWOX4.1

(MeWOX11/Manes.18G040300.1) and MeWOX4.2

(MeWOX5/Manes.05G175700.1). It is worth mentioning that identifying two orthologues in cassava per Arabidopsis protein is not an uncommon scenario, as cassava underwent a genome duplication in its evolutionary history (Prochnik et al. 2012). We next analysed the sequences of the identified proteins in close detail. Results confirmed that MeNST3.1, MeNT3.2, MeVND6, MeVND7.1 and MeVND7.2 all contain the NAC domain. MEME analysis of the NAC protein sequences revealed that MeNST3.1 and MeNST3.2 contain a motif that is not shared by any of the other cassava or Arabidopsis NAC sequences used in this study, arguing for potential NST3 differential functions between Arabidopsis and cassava (Supplementary Fig. S1). Our analyses also confirmed that the main motif in MeWOX4.1 and MeWOX4.2 is indeed the homeodomain (Supplementary Fig. S2). As well as containing the characteristic WOX homeodomain, WUS clade WOX genes (to which WOX4 belongs) contain a WUS-box motif which takes the consensus form TLELFPLH (Graaff et al. 2009). This motif is found in the Arabidopsis WOX4 sequence and in both MeWOX4.1 and MeWOX4.2 (Supplementary Fig. S2). In addition, MeWOX4.1 and MeWOX4.2 present a region in the beginning of the protein that is highly conserved between the two proteins but only partially conserved between cassava and Arabidopsis (Supplementary Fig. S2).

To support further our hypothesis that the identified proteins might be the actual orthologues of VND6, VND7, NST3 and WOX4 and based on the rationale that, according to the concept of synteny, relative positions of genes within chromosomes are, to a certain extent, conserved across species, we performed synteny analyses in the genomic regions containing the predicted orthologues of VND6, VND7, NST3 or WOX4 of relevant Malvidae species (including cassava) and Arabidopsis. Results revealed a high degree of synteny in all the analysed species (Supplementary Figs S3, S4, S5 and S6; Supplementary File S3).

In summary, phylogenetic, protein sequence and synteny analyses identified the 
potential cassava orthologues for NST3, VND6, VND7 and WOX4, which we named MeVND6, MeVND7.1, MeVND7.2, MeNST3.1, MeNST3.2, MeWOX4.1 and MeWOX4.2.

\section{Anatomical analyses of fibrous and storage roots}

To ensure that we could correlate the specific anatomical characteristics of the fibrous or the storage roots with the expression pattern of MeVND6, MeVND7.1, MeVND7.2, MeNST3.1, MeNST3.2, MeWOX4.1 and MeWOX4.2 in each of the root types, we first verified such anatomical differences in our cassava root samples. Stains with toluidine blue, phloroglucinol and potassium iodine of the fibrous and the storage root zones of our samples showed the expected differences between fibrous and storage roots in terms of xylem-type development, xylem lignification and starch accumulation (Figs. 3, 4) and, thus, confirmed the suitability of our collected material for gene expression analyses. Toluidine blue stains allowed for the observation of the structural organisation of the xylem between the two types of roots, confirming that the fibrous root zone of our samples developed high abundance of vessels and fibres and no xylem parenchyma, and the storage root zone developed almost exclusively xylem parenchyma (Fig. 3 b, c). The xylem in the fibrous root stained with a much stronger blue colour than in the storage root, probably due to stronger lignification. Indeed, we next stained fibrous and storage roots with phloroglucinol, which stains lignin red, and confirmed that fibrous roots show strong lignification in the xylem, as opposed to storage roots, displaying no lignification (Fig. 4 a, b). Finally, we verified the pattern of accumulation of starch granules in the parenchymatic xylem cells in the fibrous and the storage root by staining sections with potassium iodine solution. Potassium iodide stains starch black. As expected, the xylem parenchyma in storage roots (Fig. 4d) displayed a significantly higher signal than the fibre or vessel cells of the fibrous root (Fig. 4c). In summary, our morphological analyses verified the differences in xylem cell types development, lignification levels and starch accumulation between the fibrous root and the storage roots, confirming the suitability of our samples for gene expression analyses.

\section{Fig. 4}

Cassava root anatomy. a, b Phloroglucinol stain highlights differences in lignin content between the fibrous root and the storage root xylem. a Fibrous root xylem 
(vessels and fibres) stains red. b Storage root xylem (xylem parenchyma) does not stain. c, $\mathbf{d}$ Iodine stain highlights differences in starch content between the fibrous root and the storage root xylem. Arrows in $\mathbf{c}$ and $\mathbf{d}$ point to starch granules. All images taken at $20 \times$ magnification. Scale bar $25 \mu \mathrm{m}$

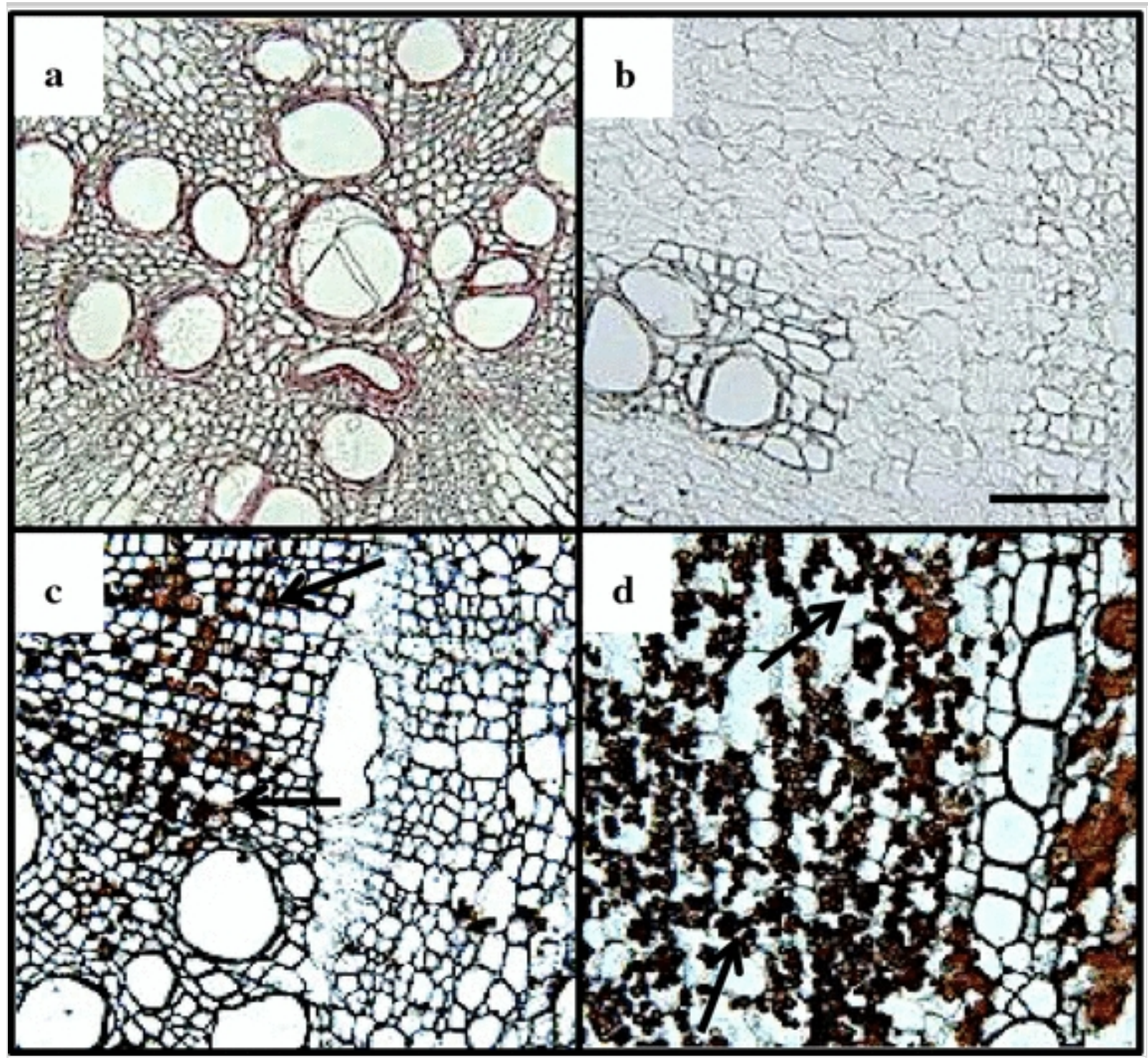

Expression of the MeVND6, MeVND7, MeNST3 and $\mathrm{MeWOX} 4$ genes in the fibrous and the storage root of cassava

Based on the rationale that MeVND6, MeVND7.1 and MeVND7.2 should conserve the Arabidopsis VND6 and VND7 function (regulating programmed cell death in the xylem), we hypothesised that the expression of MeVND6, MeVND7.1 and MeVND7.2 should be high in the fibrous roots and low or undetectable in the storage roots. This is because the xylem in the fibrous root is 
mainly formed of fibre and vessel cells (Figs. 3 b, 4a), which are lignified and dead, while the xylem parenchyma cells (the vast majority of the xylem cells in the storage roots) remain alive at maturity (Buschmann et al. 2002) and, therefore, do not undergo PCD neither are they lignified (Figs. 3c, 4b). In accordance with our hypothesis our expression analyses showed that both MeVND7.1 and MeVND7.2 are strongly expressed in fibrous roots, and not expressed at all in storage roots (Fig. 5 a). Both MeVND7.1 and MeVND7.2 displayed very similar expression levels in fibrous roots. In clear contrast, MeVND6 was expressed in both root-types, although its expression level was significantly lower than the observed for MeVND7.1 and MeVND7.2 (Fig. 5b).

\section{Fig. 5}

Gene expression analyses. a Expression analyses of VND7.1 and VND7.2. b Expression analyses of VND6. c Expression analyses of NST3.1 and NST3.2. d Expression analyses of WOX4.1 and WOX4.2. FR fibrous root, SR storage root. MeUBI: Manihot esculenta Ubiquitin 1 (reference gene)

\section{a $\mathrm{MeVND} 7$}

FR

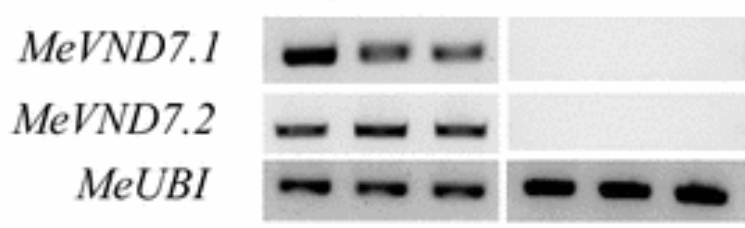

c $\mathrm{MeNST3}$

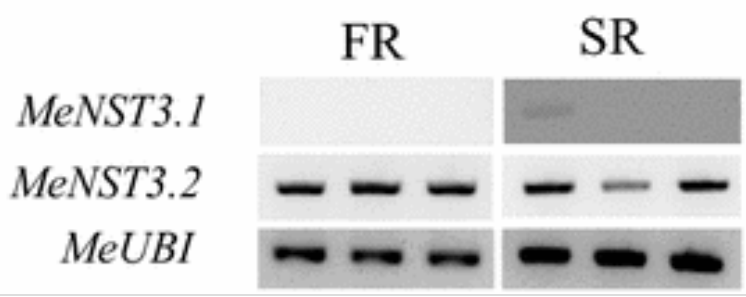

\section{b $M e V N D 6$}

FR

SR

MeVND6

$\mathrm{MeUBI}$

\section{d $\mathrm{MeWOX} 4$}

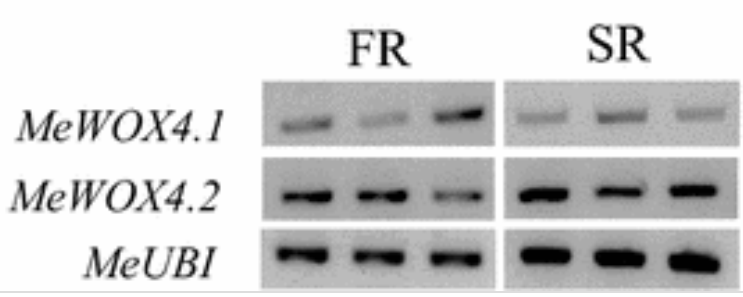

As mentioned above, NST3 regulates multiple cell wall-associated functions (i.e.: cellulose, xylan and lignin biosynthesis) by regulating the expression of a number of transcription factors in Arabidopsis (Zhong et al. 2006, 2007, 2008; Ohashi-Ito et al. 2010), and it has been suggested that such functions might be organ specific. To learn new aspects about MeNST3.1 and MeNST3.2, we tested the MeNST3.1 and MeNST3.2 expression in both the fibrous and in the storage roots of cassava. Our results showed a clear difference in terms of expression 
levels between MeNST3.1 and MeNST3.2. Indeed, MeNST3.2 showed a rather high expression level in both the fibrous and the storage roots, while MeNST3.1 expression levels were- so low (both in the fibrous and in the storage root) that we could only detect a faint reminiscent signal in one of our samples (Fig. $5 \mathrm{c}$ ).

Because xylem differentiates from the stem cells within the cambium, we questioned whether the difference in size between the fibrous and the storage roots (Figs. 1, 3 a) is directly linked with differential cambial activity between the two root types. Since in Arabidopsis WOX4 is a major regulator of cellular proliferation within the cambium, we aimed at addressing such question by understanding the expression pattern of MeWOX4.1 and MeWOX4.2 in fibrous and storage roots. Our results clearly showed that MeWOX4.2 displays higher expression levels than MeWOX4.1, both in fibrous and storage roots (Fig. $5 \mathrm{~d}$ ). Results suggest that the difference in size between the two root-types is not a consequence of MeWOX4 activity and that other factors might be involved.

\section{Discussion}

Determining the molecular basis controlling the switch from the fibrous to the storage root growth programme in cassava is a long-term challenge that holds great promise for cassava biotechnology strategies. Understanding general aspects of the expression pattern of genes (potentially) regulating xylem cell+type determination and cambial activity at the root type-specific level represents a first step towards unravelling new insights about how these two processes may participate in the switch from fibrous to storage root development.

As a first approach to identify genes potentially involved in xylem cell-type determination or cambium cellular proliferation, we used a strategy based on identifying orthologues in cassava for proteins previously known to regulate such processes in Arabidopsis. We identified MeVND6, MeVND7.1, MeVND7.2, MeNST3.1 and MeNST3.2 as the potential cassava orthologues for the Arabidopsis xylem cell-type determination regulators VND6, VND7 and NST3. We also identified MeWOX4.1 and MeWOX4.2 as the potential cassava orthologues for the Arabidopsis cambium activity regulator WOX4. Subsequently, we investigated the expression pattern of the genes encoding such proteins in samples enriched in cambium and developing xylem of the fibrous or storage roots. 
Figure 5 a shows that MeVND7.1 and MeVND7.2 are expressed in the fibrous but not in the storage root. This result is of great value because it clearly reflects that there are differential expression patterns between the fibrous and storage root, and that such differences may operate at the level of xylem differentiation (potential) regulators. Results suggest that the VND7 function may be conserved between Arabidopsis and cassava, since VND7 regulates vessel formation in Arabidopsis and, in the cassava root system, vessels are present in the fibrous but not in the storage root.

The fibrous root-specific expression of MeVND7.1 and MeVND7.2 allows for formulating new questions related not only with cassava root development but also with cassava biotechnology. For example, how would repressing the expression of MeVND7.1 and MeVND7.2 in the fibrous root affect xylem development? If MeVND7.1 and MeVND7.2 are confirmed as xylem regulators, can we alter the fibrous root-associated xylem properties? Can we induce storage root development artificially by modifying the developmental programme within the fibrous root? Further experimentation using the MeVND7.1 and/or MeVND7.2 promoters will be needed to address such questions. It is worth mentioning that, although MeVND7.1 and MeVND7.2 may also be expressed within other organs (i.e.: leaves or stem), it would still be possible to restrict transgene expression under the MeVND7.1 or MeVND7.2 promoters to the fibrous root by using inducible transgene expression approaches.

In Arabdiospsis, VND6 and VND7 are highly redundant (Zhong et al. 2006; Ohashi-Ito et al. 2010). However, our results suggest that this is not the case in the roots of cassava, since MeVND6 was expressed both in fibrous and storage roots. We speculate that MeVND7.1 and MeVND7.2 fulfil in cassava all the roles that VND6 and VND7 fulfil together in Arabidopsis. However, further experimentation is needed to understand the potential role of MeVND6, the specific function of MeVND7.1 and MeVND7.2 and the relationship between MeVND6 and the two MeVND7s during root development in cassava.

MeNST3.2 displayed high expression levels both in the fibrous and the storage roots, while MeNST3.1 was not detectable in any of the root types. Results suggest that, at least in the root context, only MeNST3.2 is functional. 
In Arabidopsis, parenchyma cells of specific organs (but not of others) develop secondary cell wall upon NST3 ectopic expression (Zhong et al. 2006). It was suggested that such effect is either due to different organs displaying differential sensitivity to NST3-mediated secondary cell wall induction or to parenchyma cells of certain organs (but not of others) being able to degrade (or inactivate) NST3 in an organ type-specific manner (Zhong et al. 2006). While both interpretations would fit with our results, we propose yet another plausible possibility in which MeNST3.2, rather than being inactivated or less sensed in storage roots' xylem parenchyma, plays different roles in the fibrous and the storage roots. Since NST3 proteins are known to regulate the expression of a number of transcription factors involved in several aspects of secondary cell wall biosynthesis (Zhong et al. 2008; Ohashi-Ito et al. 2010), we propose that the induction of certain transcription factors (but not of others) might be celltype or organ specific. In this way, the TFs which expression would be activated by NST3 in the fibrous root would be different to the ones activated in the storage roots. A potential direction in which to conduct future research based on the results reported here would be to identify the MeNST3.2 targets and test their role in xylem development within the fibrous or the storage root. Such targets do not necessarily have to be the orthologues of the NST3 targets in Arabidopsis. Indeed, Supplementary Fig. S1 shows that both MeNST3.1 and MeNST3.2 contain motifs that are not present in Arabidopsis NST3, perhaps representing an indicator of functional divergence between the two species that might lead to differential target affinity. Since MeNST3.2 is expressed in both root types, biotechnology applications aimed at manipulating simultaneously specific properties of both the fibrous or the storage roots-by using the MeNST3.2 promoter to drive transgene expression-may derive from these results.

We questioned whether the size difference between the storage and the fibrous root is linked to differential cellular proliferation within the cambium. Our expression analyses showed that there is no significant difference between the fibrous and the storage root in terms of MeWOX4.1 or MeWOX4.2 expression levels (Fig. 5 d). Straightforward interpretations of our results are (1) that the WOX4 activity may not be conserved between Arabidopsis and cassavaindeed, MeWOX4.1 and MeWOX4.2 present a domain that is not conserved in Arabidopsis, suggesting a potential functional divergence between the two 
species - or (2) that, if there is higher cambium activity in storage roots than in the fibrous root, such difference operates through factors other than MeWOX4. However, since the xylem parenchyma cells are special among the xylem celltypes in that they retain the ability to divide when they are mature (Schuetz et al. 2013), it could as well be the case that the difference in size between the fibrous and the storage roots depends (at least partly) on parenchyma xylem proliferation. All three scenarios fit with our results and further experimentation is needed to address what is the actual one.

From a biotechnology point of view, since the two MeWOX4 genes are expressed in both root-types, if the WOX4 function were conserved between Arabidopsis and cassava, enhancing the MeWOX4.1 and/or MeWOX4.2 expression should lead to a general root thickness enhancement. It is expectable that the effect would impact on both root-types, implying that it would not only be beneficial to obtain larger storage roots, but also to enhance the transport capacity of water and nutrients by enhancing the amount of xylem fibres and vessels in fibrous roots.

In conclusion, we have identified potential orthologues in cassava for pivotal regulators of xylem cell-type determination and cambium activity in Arabidopsis. Gene expression analyses revealed the expression pattern of such genes on samples enriched in cambium and developing xylem in fibrous and storage roots of cassava. Our results provide new information about cassava root development with high potential for food security-oriented breeding and biotechnology programmes.

$\mathrm{AQ} 1$

Author contribution statement TS and BC conducted experiments. JA conceived and supervised the project and wrote the manuscript.

\section{Acknowledgements}

We acknowledge John Beeching (University of Bath) for providing plants, David Alabadí (IBMCP-CSIC-UPV, Valencia), Miguel Blazquez (IBMCPCSIC-UPV, Valencia), Liam Dolan (University of Oxford) and Ana Milhinhos (University of Oxford) for critical review of the manuscript and/or scientific discussions. This work was partially supported by The Royal Society-UK 
(RG15032).

\section{Electronic supplementary material}

Below is the link to the electronic supplementary material.

Suppl. Fig. S1 MEME alignments of AtNST3, MeNST3.1, MeNST3.2 identify the NAC domain in all three sequences and a domain present in cassava but notconserved in Arabidopsis (PDF $200 \mathrm{~kb}$ )

Suppl. Fig. S2 MEME alignments of AtWOX4, MeWOX4.1, MeWOX4.2 identify the homeobox domain and the WUS-Box motif in all three sequences (PDF $204 \mathrm{~kb}$ )

Suppl. Fig. S3 Synteny between the genomic region containing VND6 putative orthologues in Manihot esculenta, Linum usitatissimum, Populus trichocarpa, Salix purpurea, Gossypium raimondii, Theobromøa cacao and Arabidopsis thaliana. Each box represents a gene. Boxes point to the gene orientation. Lines connect genes encoding the same putative orthologue across species. Dark grey central boxes represent the VND6 orthologue in each species. White boxes represent unknown proteins or proteins with no function assigned (PDF $78 \mathrm{~kb}$ )

Suppl. Fig. S4 Synteny between the genomic region containing VND7 putative orthologues in Manihot esculenta, Linum usitatissimum, Populus trichocarpa, Salix purpurea, Gossypium raimondii, Theobrom $\theta$ a cacao and Arabidopsis thaliana. Each box represents a gene. Boxes point to the gene orientation. Lines connect genes encoding the same putative orthologue across species. Dark grey central boxes represent the VND7 orthologue in each species. White boxes represent unknown proteins or proteins with no function assigned (PDF $78 \mathrm{~kb}$ )

Suppl. Fig. S5 Synteny between the genomic region containing NST3 putative orthologues in Manihot esculenta, Linum usitatissimum, Populus trichocarpa, Gossypium raimondii, Theobromøa cacao and Arabidopsis thaliana. Each box represents a gene. Boxes point to the gene orientation. Lines connect genes 
encoding the same putative orthologue across species. Dark grey central boxes represent the NST3 orthologue in each accession. White boxes represent unknown proteins or proteins with no function assigned (PDF $85 \mathrm{~kb}$ )

Suppl. Fig. S6 Synteny between the genomic region containing WOX4 putative orthologues in Manihot esculenta, Linum usitatissimum, Populus trichocarpa, Salix purpurea, Gossypium raimondii, Theobrom $\theta$ a cacao and Arabidopsis thaliana. Each box represents a gene. Boxes point to the gene orientation. Lines connect genes encoding the same putative orthologue across species. Dark grey central boxes represent the WOX4 orthologue in each species. White boxes represent unknown proteins or proteins with no function assigned (PDF $82 \mathrm{~kb}$ )

Suppl. File S1 Trimmed alignments for NAC phylogeny analyses (TXT $4 \mathrm{~kb}$ )

Suppl. File S2 Trimmed alignments for WOX phylogeny analyses (TXT $1 \mathrm{~kb}$ )

Suppl. File S3 Genes used for synteny analyses (PDF $87 \mathrm{~kb}$ )

Suppl. Table S1 Primers used for gene expression analyses (PDF 16 kb)

Suppl. Table S2 Accession numbers of proteins used for phylogeny (PDF $17 \mathrm{~kb}$ )

\section{References}

Agusti J, Merelo P, Cercos M, Tadeo FR, Talon M (2008) Ethylene-induced differential gene expression during abscission of citrus leaves. J Exp Bot 59(10):2717-2723

Agusti J, Lichtenberger R, Schwarz M, Nehlin L, Greb T (2011)

Characterization of transcriptome remodelling during cambium formation 
identifies MOL1 and RUL1 as opposing regulators of secondary growth. PLoS Genet 7(2):e1001312. doi:10.1371/journal.pgen.1001312

Altschul SF, Gish W, Miller W, Myers EW, Lipman DJ (1990) Basic local alignment search tool. J Mol Biol 215(3):403-410

Alves AAC (2002) Cassava botany and physiology. In: Hillcocks RJ, Thresh JM (eds) Cassava: biology, production and utilization. CABI, Wallingford, pp 67-89

Bailey TL, Boden M, Buske FA, Frith M, Grant CE, Clementi L, Ren J, Li WW, Noble WS (2009) MEME SUITE: tools for motif discovery and searching. Nucleic Acids Res 37:W202-W208

Buschmann H, Potter UJ, Beeching JR (2002) Ultrastructure of cassava root by TEM and SEM. Microsc Anal 87(1):9-11

Ceballos H, Kulakow P, Hershey C (2012) Cassava breeding: current status, bottlenecks and the potential of biotechnology tools. Trop Plant Biol $5(1): 73-87$

Chaweewan Y, Taylor N (2015) Anatomical assessment of root formation and tuberization in cassava (Manihot esculenta Crantz). Trop Plant Biol $8(1): 1-8$

El-Sharkawy MA (2004) Cassava biology and physiology. Plant Mol Biol 56(4):481-501

Graaff E, Laux T, Rensing SA (2009) The WUS homeobox-containing (WOX) protein family. Genome Biol 10(12):248

Guindon S, Dufayard JF, Lefort V, Anisimova M, Hordijk W, Gascuel O (2010) New algorithms and methods to estimate maximum-likelihood phylogenies: Assessing the performance of PhyML 3.0. Systematic Biol 59(3):307-321

Hirakawa Y, Kondo Y, Fukuda H (2010) TDIF peptide signaling regulates 
vascular stem cell proliferation via the $W O X 4$ homeobox gene in Arabidopsis. Plant Cell 22(8):2618-2629

Hussey SG, Saidi MN, Hefer CA, Myburg AA, Grima-Pettenati J (2015) Structural, evolutionary and functional analysis of the NAC domain protein family in Eucalyptus. New Phytol 206(4):1337-1350

Ji J, Strable J, Shimizu R, Koenig D, Sinha N, Scanlon MJ (2010) WOX4 promotes procambial development. Plant Physiol 152:1346-1356

Jones P, Binns D, Chang H-Y, Fraser M, Li W, McAnulla C, McWilliam H, Maslen J, Mitchell A, Nuka G, Pesseat S, Quinn AF, Sangrador-Vegas A, Scheremetjew M, Yong S-Y, Lopez R, Hunter S (2014) InterProScan 5: genome-scale protein function classification. Bioinformatics 30(9):12361240. doi:10.1093/bioinformatics/btu031

Katoh K, Standley DM (2013) MAFFT multiple sequence alignment software version 7: improvements in performance and usability. Mol Biol Evol 30(4):772-780

Kubo M, Udagawa M, Nishikubo N, Horiguchi G, Yamaguchi M, Ito J, Mimura T, Fukuda H, Demura T (2005) Transcription switches for protoxylem and metaxylem vessel formation. Gene Dev 19(16):1855-1860

Lebot V (2009) Tropical root and tuber crops. Cassava, sweet potato, yams and aroids. Crop production science in horticulture series (17). CAB books, CABI, Wallingford, UK

Mitsuda N, Seki M, Shinozaki K, Ohme-Takagi M (2005) The NAC transcription factors NST1 and NST2 of Arabidopsis regulate secondary wall thickening and are required for anther dehiscence. Plant Cell 17(11):29933006

Mitsuda N, Iwase A, Yamamoto H, Yoshida M, Seki M, Shinozaki K, OhmeTakagi M (2007) NAC transcription factors, NST1 and NST3, are key regulators of the formation of secondary walls in woody tissues of Arabidopsis. Plant Cell 19(1):270-280 
Nakano Y, Yamaguchi M, Endo H, Rejab NA, Ohtani M (2015) NAC-MYBbased transcriptional regulation of secondary cell wall biosynthesis in land plants. Front Plant Sci 5(6):288

Ohashi-Ito K, Oda Y, Fukuda H (2010) Arabidopsis VASCULAR-RELATED NAC-DOMAIN6 directly regulates the genes that govern programmed cell death and secondary wall formation during xylem differentiation. Plant Cell 22(10):3461-3473

Olsen AN, Ernst HA, Leggio LL, Skriver K (2005) NAC transcription factors: structurally distinct, functionally diverse. Trends Plant Sci 10(2):7987

Prochnik S, Marri PR, Desany B, Rabinowicz PD, Kodira C, Mohiuddin M, Rodriguez F, Fauquet C, Tohme J, Harkins T, Rokhsar DS, Rounsley S (2012) The cassava genome: Current progress, future directions. Trop Plant Biol 5(1):88-94

Reilly K, Bernal D, Cortes DF, Gomez-Vasquez R, Tohme J, Beeching JR (2007) Towards identifying the full set of genes expressed during cassava post-harvest physiological deterioration. Plant Mol Biol 64(1):187-203

Saithong T, Rongsirikul O, Kalapanulak S, Chiewchankaset P, Siriwat W, Netrpan S, Suksangpanomrung M, Meechai A, Cheevadhanarak S (2013) Starch biosynthesis in cassava: a genome-based pathway reconstruction and its exploitation in data integration. BMC Syst Biol 7:75

Sayre R, Beeching JR, Cahoon EB, Egesi C, Fauquet C, Fellman J, Fregene M, Gruissem W, Mallowa S, Manary M, Maziya-Dixon B, Mbanaso A, Schachtman DP, Siritunga D, Taylor N, Vanderschuren H, Zhang P (2011) The BioCassava Plus Program: biofortification of cassava for sub-Saharan Africa. Annu Rev Plant Biol 62:251-272

Schuetz M, Smith R, Ellis B (2013) Xylem tissue specification, patterning, and differentiation mechanisms. J Exp Bot 64(1):11-31

Sehr EM, Agusti J, Lehner R, Farmer EE, Schwarz M, Greb T (2010) 
Analysis of secondary growth in the Arabidopsis shoot reveals a positive role of jasmonate signaling in cambium formation. Plant J 63(5):811-822

Sojikul P, Saithong T, Kalapanulak S, Pisuttinusart N, Limsirichaikul S, Tanaka M, Utsimi Y, Sakurai T, Seki M, Narangajavana J (2015) Genomewide analysis reveals phytohormone action during cassava storage root initiation. Plant Mol Biol 88(6):531-543

Suer S, Agusti J, Sanchez P, Schwarz M, Greb T (2011) WOX4 imparts auxin responsiveness to cambium cells in Arabidopsis. Plant Cell 23(9):3247-3259

Untergasser A, Nijveen H, Rao X, Bisseling T, Geurts R, Leunissen JA (2007) Primer3Plus, an enhanced web interface to Primer3. Nucleic Acids Res 35(Web Server):W71-W74

Whankaew S, Sraphet S, Thaikert R, Boonseng O, Smith DR, Triwitayakorn K (2015) Validation of a reference gene for transcript analysis in cassava (Manihot esculenta Crantz) and its application in analysis of linamarase and $\alpha$-hydroxynitrile lyase expression at different growth stages. Afr J Biotechnol 14(9):745-751

Xu J, Duan X, Yang J, Beeching JR, Zhang P (2013) Enhanced reactive oxygen species scavenging by overproduction of superoxide dismutase and catalase delays postharvest physiological deterioration of cassava storage roots. Plant Physiol 161(3):1517-1528

Zhong R, Demura T, Ye ZH (2006) SND1, a NAC domain transcription factor, is a key regulator of secondary wall synthesis in fibers of Arabidopsis. Plant Cell 18(11):3158-3170

Zhong R, Richardson EA, Ye ZH (2007) The MYB46 transcription factor is a direct target of SND1 and regulates secondary cell wall biosynthesis in Arabidopsis. Plant Cell 19(9):2776-2791

Zhong R, Lee C, Zhou J, McCarthy RL, Ye ZH (2008) A battery of transcription factors involved in the regulation of secondary cell wall biosynthesis in Arabidopsis. Plant Cell 20(10):2763-2782 
